\title{
Frühzeitige Prävention von Adipositas und Herz-Kreislauferkrankungen
}

\author{
Nora Merker ${ }^{1}$, Nicole Wagner ${ }^{1}$, Wilhelm Kirch ${ }^{1}$, Manfred J. Müller ${ }^{2}$ \\ ${ }^{1}$ Technische Universität Dresden, Medizinische Fakultät \\ ${ }^{2}$ Christian-Albrechts-Universität Kiel
}

\section{Early prevention of obesity and cardiovascular diseases}

The prevalence of nutrition related diseases increased exponentially during the last decades. Risk factors are acquired early in life. Preventive measures include a longer duration of breast-feeding and measures of prevention and health promotion early in childhood. These measures should be undertaken by all persons having influence on the development of children.

Verbreitung von Adipositas und HerzKreislauferkrankungen

In den letzten Jahrzehnten wurde in den Industrienationen ein steter Anstieg chronischer nicht-übertragbarer Krankheiten beobachtet. Dabei gelten Adipositas, Diabetes mellitus, Herz- Kreislauferkrankungen, Hypertonie und Hypercholesterinämie aber auch einzelne neoplastische Erkrankungen (z.B. Kolonkarzinom) als zumindest anteilig Ernährungs-abhängig oder Lebensstil-assoziiert ,die durch unausgewogene (Über-)Ernährung und gleichzeitig mangelnde körperliche Aktivität erklärt werden (World Health Report, 1999; WHO Technical report Series 894. (2000) Obesity: Preventing and managing the global epidemic. WHO). Diese Krankheiten sind heute das in der ärztlichen Praxis häufigste Gesundheitsproblem. Neuere Befunde zeigen, dass die Risikofaktoren für diese Erkrankungen bereits frühzeitig im Leben erworben werden und einen nachhaltigen Einfluss im Erwachsenenalter haben. Die Häufigkeit ihres Auftretens kann deshalb wesentlich durch Lebensphasen-übergreifende Konzepte der Primär- und Sekundärprävention beeinflusst werden.

\section{Risikofaktoren bereits im Kindesalter}

Erste Ursachen der Ernährungs-abhängigen Krankheiten sind nach heutigen Erkenntnissen bereits in der embryonalen und der frühen postnatalen Entwicklung zu finden. Eine in diesem Zusammenhang bekannte Annahme ist die „Fetal Origins“ Hypothese, nach der verschiedene Konditionen, insbesondere die Ernährung des Fötus diesen für die Entwicklung chronischer Erkrankungen im Erwachse- nenalter "programmieren" (BMJ 1995; 311: 171-174; Acta Paediatr 1997; 423(Suppl.): 178-182; Am J Cli Nutr 2000; 71(Suppl.): 1344S-1352S).

So wurde beispielsweise ein Zusammenhang zwischen niedrigem Geburtsgewicht und erhöhter KHK-Mortalität (BMJ 1993; 307: 1519-1524) sowie erhöhtem Blutdruck im Erwachsenenalter (JHypertens 1996; 14: 935-941) gefunden. Eine Beziehung zwischen niedrigem Geburtsgewicht und einem veränderten Glukosemetabolismus wurde vielfach berichtet (Diabetologia 1993; 36: 62-67; BMJ 1991; 303: 1019-1022; Am J Hypertens 1996; 9: 11A). Darüber hinaus konnte gezeigt werden, dass das Geburtsgewicht umgekehrt proportional zum Risiko ist, einen Diabetes mellitus Typ 2 im Erwachsenenalter zu entwickeln (Ann Intern Med 1999; 130: 278-84). Diese Zusammenhänge bedürfen hinsichtlich ihrer Kausalität weiterer Untersuchungen (Annu Rev Nutr 2001; 21: 73-95).

Auch die Bedeutung von mütterlichen Stillpraktiken für die Entstehung verschiedener chronischer Erkrankungen wird diskutiert (Pediatr Clin North Am 2001; 48, 125-141). Es wurde berichtet, dass Kinder, die länger gestillt wurden, im Vergleich zu solchen, die nur für kurze Zeit gestillt wurden, ein geringeres Risiko hatten, Übergewicht zu entwickeln (JAMA 2001; 285: 2461-2467). Diese Zusammenhänge wurden durch eine große Untersuchung in Bayern bestätigt (BMJ 1999; 319: 147-150; Monatsschr Kinderheilkd 149; 2001: 11). Die Auswertung der Daten dieser Studie ergab eine inversive Korrelation zwischen der Prävalenz von Übergewicht bzw. Adipositas und vorausgegangenem Stillen: $\mathrm{Zu}-$ nehmende Stilldauer senkte die Adipositas-Prävalenz. Bei Kindern, die mindestens ein halbes Jahr gestillt worden waren, nahm das Risiko für die Entstehung von Übergewicht um wenigstens 30\%, für Adipositas um über $40 \%$ ab.

In der BOGALUSA Heart Study, einer epidemiologischen Langzeit-Untersuchung in den USA, wurde festgestellt dass kardiovaskuläre Risikofaktoren inklusive des Metabolischen Syndroms bereits bei Kindern und Jugendlichen auftreten ( $\mathrm{N}$ Engl J Med 1998; 228: 1650-1656; Am J Med Sci (2001; 322: 267-274). Die Adipositas als eigenständige Entität und gleichzeitiger Risikofaktor für kardiovaskuläre Erkrankungen ist auch in Deutschland unter Kindern und Jugendlichen weit verbreitet (J Clin Epidemiol 1999; 52: 791-800). In einer Studie in Kiel wurde für die Gruppe der 5 bis 7-Jährigen eine Prävalenz des Übergewichtes von 23\% nachgewiesen (Dtsch Ärztebl 1998; 95: A2027-A2030).

Das frühzeitige Auftreten kardiovaskulärer Risikofaktoren bedeutet ein nachhaltiges Risiko für die Entwicklung von Herz-Kreislauferkrankungen im Erwachsenenalter (Int J Obes 1997; 21: 507-526). Das Risiko besteht auch fort, wenn die Betroffenen als Erwachsene Risikofaktoren eliminieren: Ein „dickes“ Kind weist auch nach Normalisierung des Körpergewichtes im Erwachsenenalter im Vergleich zu einem „dünnen“ Kind, das lebenslang „schlank“ bleibt, eine erhöhte Prädisposition für Stoffwechselerkrankungen auf (Am J Coll Cardiol 1996; 27, 277-284; Obes Rev 2001; 2: 15-28).

\section{Maßnahmen zur Prävention}

Mit diesen Erkenntnissen wird deutlich, dass präventive Maßnahmen bereits in den frühen Entwicklungsphasen von Kindern 
ergriffen werden müssen. Dabei sollte Wert auf primär- und sekundärpräventive Maßnahmen gelegt werden. Die auf das Individuum bezogene Vorsorge muss um Public Health-Strategien erweitert werden (Münch Med Wschr 2001; 143: 863-867).

Um Präventionsmaßnahmen betreiben zu können, muss zunächst definiert werden, ab welchem Punkt von Übergewicht bzw. Adipositas bei Kindern gesprochen wird. Bisher gibt es jedoch noch keine vereinheitlichte Definition zur Erhebung (Eur J Nutr 2002; 41: 61-67). Von der Arbeitsgemeinschaft „Adipositas im Kindes- und Jugendalter“ (AGA) wurden unter Einbeziehung bereits durchgeführter Untersuchungen in verschiedenen Regionen Deutschlands Perzentile für den Body-Mass-Index (BMI ) erstellt, die als Referenzwerte für die Definition von Übergewicht und Adipositas bei Kindern und Jugendlichen verwendet werden sollen (Monatsschr Kinderheilkd 2001; 149: 807-818). Bei der Berechnung dieser Werte wurden die geschlechts- und altersspezifischen Modifikationen, denen der BMI im Kindes- und Jugendalter unterliegt, berücksichtigt. Aufgrund dieser Veränderungen ist es nicht möglich, einen für das gesamte Kindes- und Jugendalter gültigen Referenzwert anzugeben. Die AGA empfiehlt, das 90. bzw. 97. Perzentil für die Definition von Übergewicht bzw. Adipositas. Die Referenzwerte für Kinder von 0-18 Jahren sind in Tab. 1 zu finden.

Der Wert bevölkerungsbezogener Präventionsmaßnahmen ist am Beispiel von Herz-Kreislauferkrankungen gut belegt (Prev Med 1996; 25: 243-250; Prev Med 1996; 25: 135-145). In den USA wurde auf der Grundlage der Bogalusa Heart Study, eine große Anzahl Gesundheitsförderungsprogramme für das Schulsetting in verschiedenen Bundesstaaten entwickelt, z.B. das
Health Ahead/Heart Smart Programm (J Nutr Educ 1988:20:128-132), Gimme 5 (Health Education Behavior 2000; 27: 201-212), das Minnesota Heart Health Program (Am J Public Health 1994; 84, 1383-1393) sowie CATCH (Multicenter Child and Adolescent Trial for Cardiovascular Health) (J Sch Health 1990; 60: 406-413). Sämtliche dieser Interventionsprogramme beinhalten Ernährung, als Bestandteil gesunder Lebensführung in ihren Curricula.

In Deutschland sind in den vergangenen zwei Jahrzehnten eine Reihe von Programmen zur Prävention und Gesundheitsförderung im Bereich Ernährung entstanden, die auf verschiedene Zielgruppen ausgerichtet sind. Dazu gehören u.a. die Kieler Adipositaspräventionsstudie (Kiel Obesity Prevention Study; KOPS), die als Zielgruppe sowohl gesunde Kinder als auch Kinder mit definiertem Risiko hat. Im Rahmen von KOPS wird seit 1996 als Intervention Ernährungserziehung in Schulen (für bisher 910 Kinder) kombiniert mit Familienberatung (in 92 Familien) durchgeführt (Akt Ernährungsmed 2000; 25: 33-37; Obes Rev 2001; 2: 15-28). Erste Ergebnisse der 1- und auch der 4-Jahres-Nachbeobachtung zeigen eine bessere Entwicklung des Ernährungszustandes und auch eine geringere Inzidenz des Übergewichtes nach Schulintervention. Bereits übergewichtige Kinder profitieren von einer Familienintervention.

Ferner läuft seit nunmehr 7 Jahren in Nürnberg das Präventionserziehungsprogramm PEP für Familien (J Clin Epidemiol 1999; 52: 791-800). PEP zeigt laut einer Familienbefragung, die im Jahr 1999 zum ersten Mal und in 2000 wiederholt wurde, gute Verhaltensänderungen (Essen und Bewegung) sowie einen Informationsgewinn für alle Betroffenen.

Ebenfalls eine Maßnahme der Gesundheitsförderung ist die „Ernährungserziehung Sachsen“, die auf Vor- und Grundschulkinder abzielt, ohne explizit den Risikostatus zu berücksichtigen. Für diese Aktivität konnten Erfolge für ernährungsbezogene Wissensinhalte, Einstellungen und Verhaltensweisen gezeigt werden (Z Pädagog Psychol 2002; 16: 43-50).
Tab. 1 Referenzwerte des BMI (in kg/m²) zur Definition von Übergewicht (90. Perzentil) und von Adipositas (97. Perz.).

\begin{tabular}{lllll}
\hline & \multicolumn{2}{c}{ Jungen } & \multicolumn{2}{c}{ Mädchen } \\
Alter & 90. & 97. & 90. & 97. \\
(Jahre & Perzentil & Perzentil & Perzentil & Perzentil \\
\hline 0 & 14,28 & 15,01 & 14,12 & 14,81 \\
\hline 1 & 18,73 & 19,81 & 18,25 & 19,22 \\
2 & 18,01 & 19,14 & 17,92 & 19,03 \\
3 & 17,62 & 18,82 & 17,64 & 18,84 \\
4 & 17,54 & 18,83 & 17,54 & 18,85 \\
5 & 17,61 & 19,02 & 17,69 & 19,16 \\
\hline 6 & 17,86 & 19,44 & 17,99 & 19,67 \\
7 & 18,34 & 20,15 & 18,51 & 20,44 \\
8 & 19,01 & 21,11 & 19,25 & 21,47 \\
9 & 19,78 & 22,21 & 20,04 & 22,54 \\
10 & 20,60 & 23,35 & 20,80 & 23,54 \\
11 & 21,43 & 24,45 & 21,61 & 24,51 \\
\hline 12 & 22,25 & 25,44 & 22,48 & 25,47 \\
13 & 23,01 & 26,28 & 23,33 & 26,33 \\
\hline 14 & 23,72 & 26,97 & 24,05 & 27,01 \\
\hline 15 & 24,36 & 27,53 & 24,59 & 27,45 \\
16 & 24,92 & 27,99 & 24,91 & 27,65 \\
17 & 25,44 & 28,40 & 25,11 & 27,72 \\
18 & 25,91 & 28,78 & 25,28 & 27,76 \\
\hline
\end{tabular}

Eine sekundärpräventive Maßnahme, die bei Kindern, die bereits adipös sind, durchgeführt wird, ist der Freiburg Intervention Trial for Obese Children (FITOC). FITOC besteht aus regelmäßigem körperlichen Training und einer umfassenden Ernährungsund Verhaltenserziehung (Z Ärztl Fortbild Qualitätssich 2000; 94: 677-681).

Zwei der Programme sind in Tab. 2 beschrieben. Diese haben trotz der verschiedenen Zielgruppen ein gemeinsames Anliegen: Zunahme des Konsums von Obst und Gemüse, Abnahme des Verzehrs von Fett, Steigerung der körperlichen Aktivität sowie Verringerung der Inaktivität(d.h. des Fernsehkonsums). Im Internet unter www.thieme-connect.de ist eine Reihe von für unterschiedliche Zielgruppen angebotene Präventionsprogramme in verschiedenen Regionen der BRD mit der jeweiligen Kontaktadresse aufgelistet (ohne Anspruch auf Vollständigkeit). Es existieren noch viele andere z.B. durch Krankenkassen initiierte Programme.

Bis jetzt existieren nur vereinzelte Studien, die zur Wirksamkeit von Maßnahmen zur Adipositas-Prävention durchgeführt worden sind. Laut Leitlinien zur Therapie der Adipositas der AGA (Lauterbach, Westenhöfer, Wirth, Hauner: Adipositas Leitlinie - 
Tab. 2 Vorgehen und Erfolge von zwei Gesundheitsförderungs- bzw. Präventionsprogrammen.

\begin{tabular}{|c|c|c|}
\hline Projekt & Vorgehensweisen & Ergebnisse \\
\hline \multirow[t]{2}{*}{$\begin{array}{l}\text { KOPS } \\
\text { (längerfristige } \\
\text { Intervention) }\end{array}$} & $\begin{array}{l}\text { Schulintervention für alle Kinder: Ernährungserziehung, } \\
\text { aktive Pausengestaltung, Elternabende, Weiterbildung } \\
\text { für die Lehrer über einen längeren Zeitraum }\end{array}$ & $\begin{array}{l}\text {, Verbesserung im Ernährungswissen } \\
\text { Verbesserungen im Verhalten: (Erhö- } \\
\text { hung der körperlichen Aktivität) }\end{array}$ \\
\hline & $\begin{array}{l}\text { Familienintervention für Familien mit adipösen Kin- } \\
\text { dern: individuelle Beratung zu Ernährung, körperlicher } \\
\text { Aktivität, Verhalten, Angebot eines Sportprogramms } \\
\text { www.uni-kiel.de/nutrfoodsc/nutrition/index.htm }\end{array}$ & $\begin{array}{l}\text { Verbesserungen im Verhalten: } \\
\text { Erhöhung des Obst- und Gemüse- } \\
\text { konsums, Erhöhung der körperli- } \\
\text { chen Aktivität }\end{array}$ \\
\hline $\begin{array}{l}\text { Ernährungs- } \\
\text { erziehung } \\
\text { Sachsen } \\
\text { (einmalige } \\
\text { Intervention) }\end{array}$ & $\begin{array}{l}\text { Vermittlung theoretischen Ernährungswissens und } \\
\text { praktische Übungen durch Fachfrauen für Kinderernäh- } \\
\text { rung an Vor- und Grundschulkinder, ihre Eltern und } \\
\text { Lehrer/Erzieher, im Rahmen von Unterrichts- und } \\
\text { Projekteinheiten; derzeit noch ohne systematische } \\
\text { Begleitung der Kinder über einen längeren Zeitraum }\end{array}$ & $\begin{array}{l}\text { mittelfristige Verbesserungen in } \\
\text { ernährungsbezogenen Wissensinhal- } \\
\text { ten und Einstellungen der Kinder } \\
\text { geringfügige, kurzfristige und sehr } \\
\text { spezifische Verbesserungen im } \\
\text { Verhalten }\end{array}$ \\
\hline & v.dge.de/Pages/navigation/sektionen/Sachsen/sacl & hsen1.html \\
\hline
\end{tabular}

Tab. 3 Präventions- und Therapiemöglichkeiten auf verschiedenen Ebenen.

\begin{tabular}{|c|c|c|}
\hline Ebene & Maßnahme & Zielgruppen \\
\hline 1. Schule & $\begin{array}{l}\text { - mehr Schulsport } \\
\text { - „Bewegte Pause“ } \\
\text { - Ernährungserziehung } \\
\text { - Lebensmittelangebot }\end{array}$ & $\begin{array}{l}\text { Kinder und } \\
\text { Lehrer }\end{array}$ \\
\hline 2. Familie & $\begin{array}{l}\text { - mehr Aktivität/mehr Sport } \\
\text { - weniger Inaktivität } \\
\text { - gesunde Ernährung } \\
\text { - weniger Zwischenmahlzeiten }\end{array}$ & $\begin{array}{l}\text { Eltern und } \\
\text { Kinder }\end{array}$ \\
\hline 3. Sportverein & - mehr Angebote für „dicke Kinder“ & $\begin{array}{l}\text { Eltern und } \\
\text { Kinder }\end{array}$ \\
\hline 4. Stadt/Region & - mehr Anreize für körperliche Aktivität und gesunde Ernährung & \\
\hline $\begin{array}{l}\text { 5. Arztpraxis, } \\
\text { Ärzte im Öffentli- } \\
\text { chen Gesund- } \\
\text { heitsdienst, } \\
\text { Betriebsärzte }\end{array}$ & $\begin{array}{l}\text { - regelmäßiges Screening } \\
\text { - Anamnese und Klassifizierung von Übergewicht und Adipositas } \\
\text { - Identifizieren von Risikopersonen } \\
\text { - Identifizieren von Komorbiditäten } \\
\text { - Risikofaktoren-Management (Kardiovaskuläre Rf., F. für Gewichtszunahme) } \\
\text { - Aufklärung/Wissensvermittlung für Eltern und Kinder (Bedeutung v. Still } \\
\text { praktiken, körperlicher Aktivität, Energiezufuhr, Lebensstilveränderung } \\
\text { - Überweisung zu spezieller Adipositas-Therapie } \\
\text { - Gewichtsstabilisierung }\end{array}$ & $\begin{array}{l}\text { Eltern und } \\
\text { Kinder } \\
\text { ) }\end{array}$ \\
\hline
\end{tabular}

Evidenzbasierte Leitlinie zur Behandlung der Adipositas in Deutschland, Heinrich-Heine-Universität Düsseldorf 1998) besteht Evidenz, dass Kinder eine Hauptzielgruppe für die Prävention von Adipositas sein sollten. Eine Studie konnte belegen, dass insbesondere unter Einbeziehung der Eltern in solche Programme nachhaltige Effekte in der Gewichtsreduktion erzielt werden können (JAMA 1990; 264: 2519-2523). Somit ist es wichtig, alle Personen, die Einfluss auf die frühkindliche und spätere Entwicklung von Kindern und Jugendlichen haben, in die präventiven Maßnahmen einzubeziehen. Aus ärztlicher Sicht reicht die Spanne der Verantwortlichen vom Geburtshelfer bzw. Gynäkologen bis hin zu Allgemein-, Kinder-, Jugend- und Schulärzten. Aus Sicht von Public Health müssen in die Prävention auch die kommunale und politische Ebene ein- bezogen werden, um auf alle Beteiligten einen Effekt zu haben.

Fazit

Präventionsmaßnahmen sind eine Herausforderung für verschiedenste Bereiche (Tab. 3). Dabei vertreten die praktischen Ärzte eine derzeit noch zu wenig vordergründige Handlungsebene.

Möglichkeiten für den praktizierenden Arzt, präventive Arbeit zu leisten, liegen zum einen darin, Frauen über die Bedeutung aufzuklären, die das Stillen für die Entwicklung von Adipositas bei Kindern hat. Zum anderen können durch Screening-Maßnahmen Kinder mit Risikofaktoren identifiziert und $\mathrm{zu}$ entsprechenden Präventionsmaßnahmen geführt werden. Darüber hinaus müssen Eltern über die Zu- sammenhänge zwischen der Entstehung von Übergewicht sowie anderen kardiovaskulären Risikofaktoren beim Kind und Gesundheit im späteren Lebensalter aufgeklärt werden, um diese aktiv in Präventionsmaßnahmen einzubeziehen. Zudem sind Ärzte aufgefordert, sich aktiv in Präventionsprogramme, die beispielsweise in Schulen initiiert werden, einzubringen.

Eine wichtige von allen Beteiligten zu erbringende Leistung ist, das derzeit vorliegende Wissen zu bündeln und eine einheitliche Grundlage bei der Prävention bzw. Therapie von Adipositas zu verwenden. Die Basis dafür kann in den Leitlinien zur Therapie und Prävention der Adipositas im Kindes- und Jugendalter der AGA gefunden werden (Konsensus-Konferenz der AGA am 19.10.2001 in Bremen, Wabitsch \& Kunze für die AGA).

Korrespondenz

Dipl.-Psych. Nora Merker

Prof. Dr. Dr. Wilhelm Kirch Technische Universität Dresden,

Medizinische Fakultät BMBF-Forschungsverbund Public Health Sachsen

Fiedlerstraße 33 01307 Dresden Tel: 0351/4583254

\section{Fachliche Betreuung der "Serie Prävention“":}

Prof. Dr. Dr. h. c. Peter C. Scriba Medizinische Klinik / Klinikum Innenstadt der Universität München

Ziemssenstr. 1

80336 München

Prof. Dr. Friedrich W. Schwartz Medizinische Hochschule Hannover Abteilung für Epidemiologie, Sozialmedizin und Gesundheitsforschung

Carl-Neuberg-Str. 1

30623 Hannover 